\title{
The incidence of Type I diabetes has not increased but shifted to a younger age at diagnosis in the 0-34 years group in Sweden 1983 to 1998
}

\author{
A. Pundziute-Lyckå1, G. Dahlquist' ${ }^{1}$, L. Nyström² ${ }^{2}$ H. Arnqvist ${ }^{3}$, E. Björk ${ }^{4}$, G. Blohmé5, J. Bolinder ${ }^{5}$, \\ J. W. Eriksson ${ }^{2}$, G. Sundkvist ${ }^{6}$, J. Östman ${ }^{7}$, and the Swedish Childhood Diabetes Study Group \\ ${ }^{1}$ Department of Clinical Sciences, Paediatrics, ${ }^{2}$ Department of Public Health and Clinical Medicine, Epidemiology and Medicine, \\ Umeå University, Umeå, Sweden \\ ${ }^{3}$ Department of Medicine and Care, Linköping University, Linköping, Sweden \\ ${ }^{4}$ Department of Internal Medicine, University Hospital, Uppsala, Sweden \\ ${ }^{5}$ Department of Medicine, Karolinska Institute, Söder Hospital and Huddinge University Hospital, Stockholm, Sweden \\ ${ }^{6}$ Department of Endocrinology, Malmö University Hospital, Malmö, Sweden \\ ${ }^{7}$ Center of Metabolism and Endocrinology, Karolinska Institute, Huddinge University Hospital, Stockholm, Sweden
}

\begin{abstract}
Aims/hypothesis. To analyse the incidence of Type I (insulin-dependent) diabetes mellitus in the 0-34 years age group in Sweden 1983-1998.

Methods. Incidence and cumulative incidence per 100000 and Poisson regression analysis of age-period effects was carried out using 11751 cases from two nation-wide prospective registers.

Results. Incidence (95\%-CI) was 21.4 (20.8-21.9) in men and 17.1 (16.6-17.5) in women between 0 and 34 years of age. In boys aged $0-14$ and girls aged 0-12 years the incidence increased over time, but it tended to decrease at older age groups, especially in men. Average cumulative incidence at 35 years was 748 in men and 598 in women. Cumulative incidence in men was rather stable during four 4-year periods $(736,732,762,756)$, while in women it varied more $(592,542,617,631)$. In males aged 0-34 years, the
\end{abstract}

incidence did not vary between the 4-year periods ( $p=0.63)$, but time changes among the 3 -year age groups differed $(p<0.001)$. In females the incidence between the periods varied $(p<0.001)$, being lower in 1987-1990 compared to 1983-1986, but time changes in the age groups did not differ $(p=0.08)$. For both sexes median age at diagnosis was higher in 1983-1986 than in $1995-1998(p<0.001) \quad(15.0$ and 12.5 years in males; 11.9 and 10.4 in females, respectively).

Conclusion/interpretation. During a 16-year period the incidence of Type I diabetes did not increase in the 0-34 years age group in Sweden, while median age at diagnosis decreased. A shift to younger age at diagnosis seems to explain the increasing incidence of childhood Type I diabetes. [Diabetologia (2002) 45:783-791]

Keywords Type I diabetes mellitus, incidence, secular trend, epidemiology.
Received: 19 November 2001 / Revised: 6 February 2002 Published online: 8 May 2002

(C) Springer-Verlag 2002

Corresponding author: Dr. A. Pundziute-Lyckå, Department of Clinical Sciences, Paediatrics, Umeå University, 90785 Umeå, Sweden, e-mail: auste.pundziute.lycka@pediatri.umu.se

Abbreviations: DISS, Diabetes Incidence Study in Sweden; LADA, latent autoimmune diabetes in adults; ICA, islet cell auto-antibody; IA-2A, thyrosine phosphatase auto-antibody
The current theory is that Type I (insulin-dependent) diabetes mellitus develops in genetically susceptible individuals as a result of progressive autoimmune destruction of beta cells and environmental factors are believed to be involved both as triggers, modifiers and promoters of the disease occurrence $[1,2,3,4,5]$. Most epidemiological studies on the incidence of Type I diabetes have been performed in 0-14 years old children, due to ease of case ascertainment and straightforward classification. However, a large proportion of Type I diabetes cases are diagnosed later in life $[6,7$, $8,9]$. 
During the last decades, an increase of childhood Type I diabetes incidence has occurred in many countries worldwide [10, 11], in Europe [12] and in Sweden [13]. However, little is known about the time trend in a broader age span [14, 15], as most of the registers covering diabetes incidence above the age of 14 years have been operating for a relatively short period of time.

Analysis of the variation of incidence over time could shed light on the causes of Type I diabetes, because changes in incidence, which can be described as age, calendar period or birth cohort effects, can be expected to reflect the temporal changes of the population exposure to environmental risk factors. In this report, we analysed a large data set containing 11751 cases of Type I diabetes, prospectively registered over a period of sixteen years in two Swedish populationbased diabetes registers, covering the age group 0 to 34 years. We studied the change of cumulative incidence by the age of 35 years over time, and used Poisson regression modelling to study the effects of age and time period. Age-specific incidence was also compared among people born during the 1990s, 1980s, 1970s, 1960s and 1950s.

\section{Subjects and methods}

Age group 0-14 years: Swedish Childhood Diabetes Study $(S C D S)$. In the Swedish health care system all children aged 0-14 years with suspected diabetes are referred to paediatric departments. Since 1 July 1977, all 43 paediatric clinics in Sweden report newly diagnosed insulin-treated diabetes patients using a special form. This includes information about the patient's personal identification number, sex, county of residence, date of diagnosis (date when first insulin injection was given), date of reporting, reporting hospital and physician. Every 6 to 12 months the central register in Umeå requests the local contact person to verify and complete details about the recorded cases through the hospital medical records. The same methods of data collection and verification have been used since the start of the register. Comparisons with the registers of the Swedish Diabetes Association [16] and the Swedish Military Conscripts [17] showed that the Swedish Childhood Diabetes Register covered 96-99\% of all cases 0-14 years old at diagnosis. During the sixteen-year period (1983 through 1998), 3664 boys and 3385 girls below the age of 15 years when diabetes was diagnosed were registered.

Age group 15-34 years: Diabetes Incidence Study in Sweden (DISS). Since 1 January 1983, all departments of internal medicine $(n=96)$, paediatrics $(n=43)$, endocrinology $(n=3)$ and more than 700 primary health care units in Sweden report newly diagnosed patients with diabetes mellitus in the age group 15-34 years. Information about personal identification number, name, address, sex, date of diagnosis, physician's clinical classification of diabetes type (Type I, Type II, secondary, type unknown or not yet classified), date of reporting, reporting unit and physician and some clinical characteristics are filled in by the physician on a special form. Diabetes was diagnosed and classified according to clinical criteria as recommended by the World Health Organisation (WHO) $[18,19]$ as described in detail previously [20]. The actual classification into diabetes types was left to the physician's own judgement, based on the clinical impression at the time of diagnosis. According to common practice, severe hyperglycaemia, ketosis, low or normal body weight and an immediate need of insulin therapy are synonymous with Type I diabetes. Once a year the units who had reported at least one patient during the last year receive a list of reported patients, and every unit receives a list of patients reported since the start of the register in 1983.

During the first five years of the study (1983-1987), the completeness of ascertainment in the DISS was estimated in the two southernmost counties, covering $9.2 \%$ of the population aged 15-34 years by using a computer-based patient administrative register as a second source. The completeness of ascertainment was similar for males and females, being $86 \%$ for Type I diabetes [21]. A study in the county of Västerbotten in the Northern Sweden, covering $2.9 \%$ of the population and using a similar second source, found no obvious trend in the number of cases not reported to the DISS during 1986-1997. The completeness of ascertainment was $91 \%$ during the whole period, and during 1986-1991 and 1992-1997 it was $97 \%$ and $86 \%$, respectively.

During the four 4-year periods of the study (1983-1986, 1987-1990, 1991-1994 and 1995-1998) 71.6\%, 73.9\%, 74.9\% and $74.6 \%$ of all cases in the DISS were classified as Type I diabetes by the reporting physicians. The corresponding figures for cases which could not be classified as either Type I or Type II diabetes on clinical grounds at the time of diagnosis was $6.9 \%, 10.7 \%, 8.9 \%$ and $7.6 \%$, respectively. The proportions of Type I, Type II and unclassified diabetes cases over time were similar in males and females. A total of 3052 male and 1650 female Type I diabetes cases were recorded 1983 through 1998 in the 15-34 years age group in Sweden.

Cases diagnosed with Type I diabetes between 1 January 1983 and 31 December 1998 were selected from both registers and merged into one data file, forming a large data-set of 6716 males and 5035 females below 35 years of age at diagnosis. To calculate the age-specific incidence rates according to the birth cohort, we have also included the 0-14 year old cases recorded in the Swedish Childhood Diabetes Study between 1 January 1978 and 31 December $1982(n=1843)$. Population data were obtained from the Statistics Sweden.

Statistical methods. Age and sex specific incidence rates per 100000 and year, and cumulative incidence per 100000 were calculated. 95\%-CI for the incidence rates were estimated assuming Poisson distribution of the cases. Direct age-standardisation of the incidence rates was performed assuming a standard population with equally sized five-year age groups. Poisson regression analysis of age-period effects was done using Egret for Windows (CYTEL, Cambridge, Mass., USA). For the Poisson regression analyses age was categorised into twelve 3year age groups and calendar time into four 4-year periods (1983-1986, 1987-1990, 1991-1994, 1995-1998). Boys, 0-2 years age group and 1983-1986 years calendar period were used as reference categories. Median age at diagnosis during the 4-year calendar periods was compared using the MannWhitney test, as age at diagnosis was non-normally distributed.

In our material, the patients were born between 1948 and 1998 (0-14 years old between 1963 and 1998, 15-34 years old between 1948 and 1983). We calculated the age-specific incidence rates for 100000 live births for five successive 10-year birth cohorts - people born in the 1950s, 1960s, 1970s, 1980s and 1990s. For people born during different time periods full data was available for varying age spans at diabetes diagnosis (1950-1959: 25-34 years; 1960-1969: 10-34 years; 1970-1979: 0-26 years; 1980-1989: 0-16 years; 1990-1997: 0-6 years). Every age category in the birth cohorts covered an interval from 2 to 10 calendar birth years. 


\section{Results}

Incidence. Between 1983 and 1998, the average age standardized incidence in the 0-34 years group was significantly higher in Swedish males, 21.4 per 100000 and year (95\%-CI 20.8-21.9) than in females, 17.1 (95\%-CI 16.6-17.5). The age distribution of the incidence for males and females 1983 through 1998 is presented in Figure 1. The incidence was low in children under one year of age (only 51 cases were diagnosed during the 16-year study period), but was already high in one to two year old children. The age-specific incidence pattern was similar for males and females. However, in females the incidence peak occurred about two years earlier and the incidence decreased and remained lower than in males afterwards. In children below the age of 15 years at diagnosis, the incidence of Type I diabetes did not differ between boys and girls, while in the older age groups it was significantly higher for the males (Table 1 ).

Figure 2 shows the age-specific incidence rates per 100000 and year in 2-year age groups of males (A) and females (B) during four 4-year periods 1983 through 1998. A gradual increase over time occurred in boys aged up to 13-14 years and in girls aged up to 11-12 years at diabetes diagnosis, although the age of peak incidence appeared little changed. On the contrary, in the older age groups the incidence tended to decrease over time and was lower during the last 4-year period compared to the first one, especially in

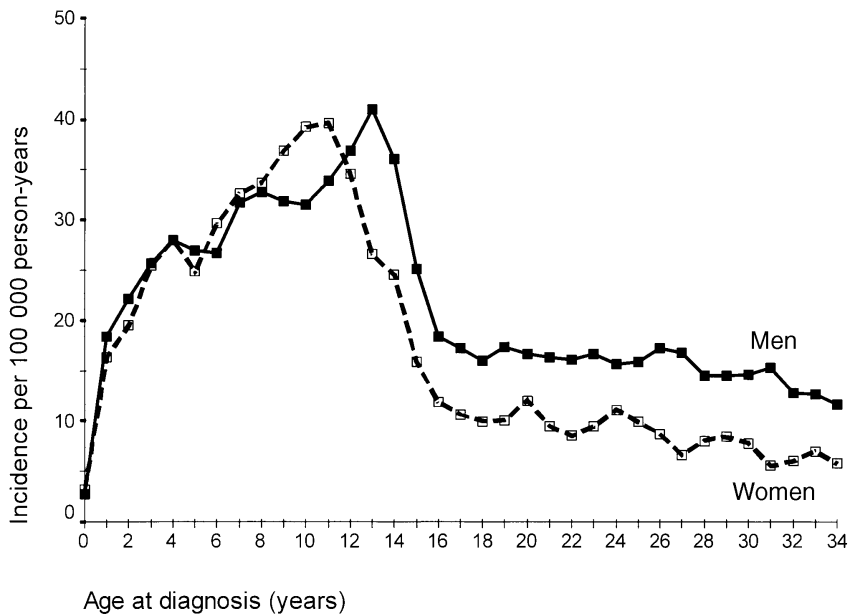

Fig. 1. Incidence of Type I diabetes per 100000 and year in 0-34 years old Swedish males and females by age 1983-1998

males (Fig. 2). Compared to 1983-1986 the ageadjusted incidence in the $0-14$ years group in $1995-1998$ was $23 \%(p<0.001)$ and $16 \%(p=0.002)$ higher in boys and girls, respectively (Table 1 ). In the 15-34 years group during the corresponding periods the age-adjusted incidence was 20\% $(p<0.001)$ lower in males, although in females the decrease was statistically not significant $(12 \%(p=0.07))$ (Table 1$)$.

Cumulative incidence. Figure 3 shows cumulative incidence curves up to the age of 35 years for males and females 1983 through 1998. By 15 years of age cumu-

Table 1. Number of Type I diabetes cases and mean incidence per 100000 and year by age group and sex in the 0-34 years group in Sweden during four 4-year periods 1983-1998

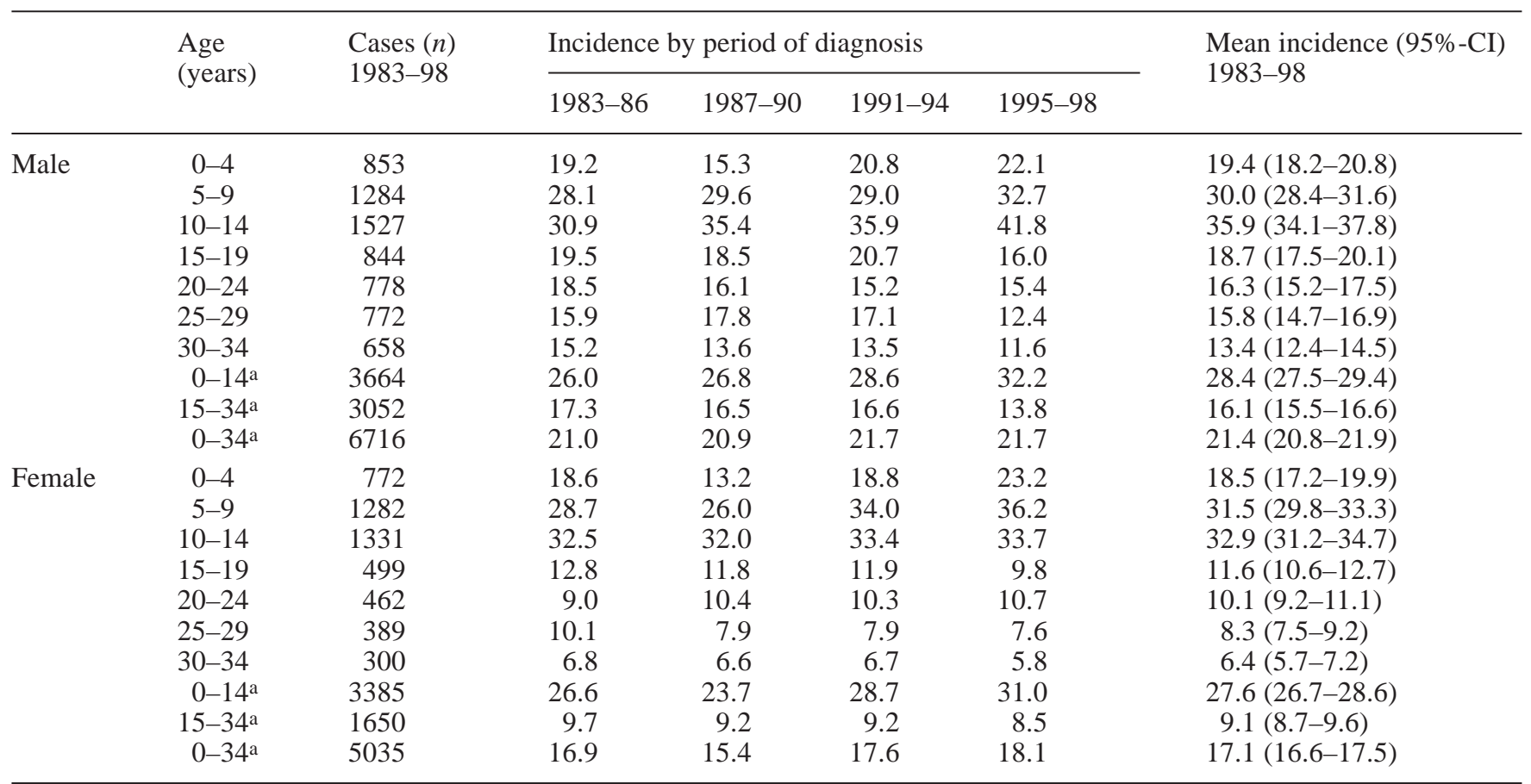

a Age standardized rates 


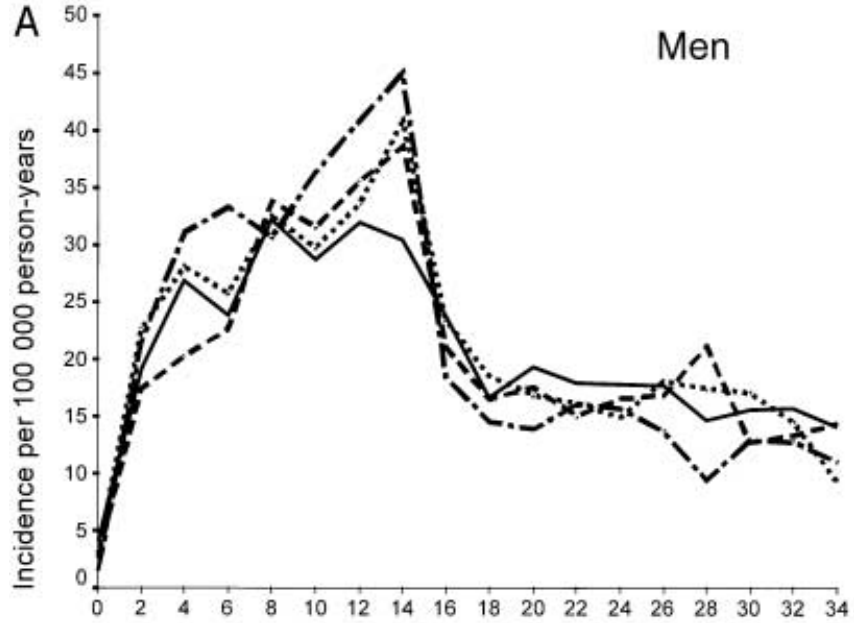

Age at diagnosis (years)

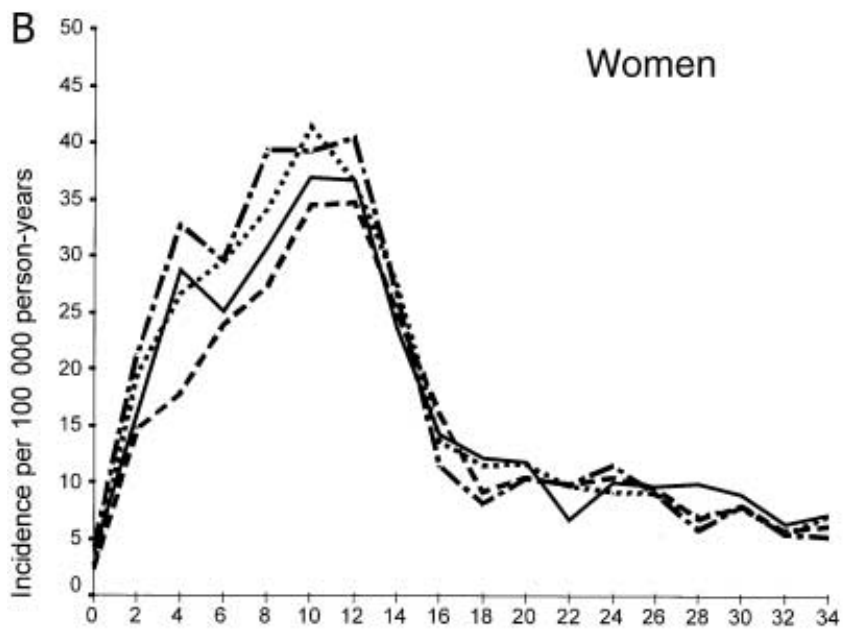

Age at diagnosis (years)

Fig. 2A, B. Incidence of Type I diabetes per 100000 and year in 0-34 years old Swedish males (A) and females (B) by age (2-year groups) during four 4-years periods 1983-1998. 1983-86 (-); 1987-90 (- - -); 1991-94 (…..); 1995-98 $(-\cdot \cdot \cdot)$

lative incidence was similar for males and females (426 and 415 per 100000 , respectively), while by 35 years of age, it was much higher for males (748 and 598 per 100000 for males and females, respectively). Figure 4 shows the cumulative incidence curves up to the age of 35 years for males and females during four 4-year periods 1983 through 1998. In males the cumulative incidence per 100000 by 15 years of age gradually increased (390, 402, 429 and $479)$, while it was rather stable by 35 years of age (736, 732, 762 and 756). During the last 4-year period (1995-1998), the increase in the cumulative incidence by 15 years of age coincided with a decrease of incidence in the older age groups, thus cumulative incidence did not increase further by 35 years of age. In

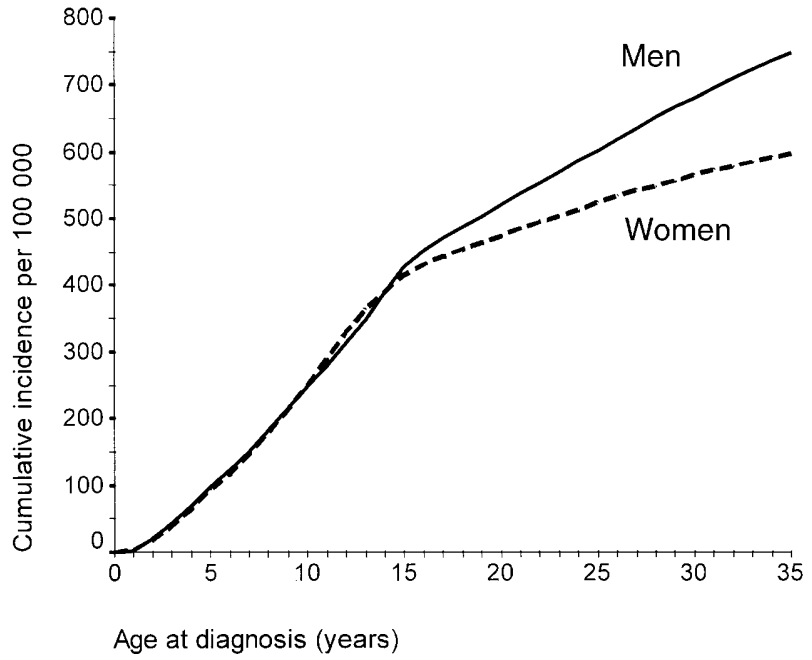

Fig. 3. Cumulative incidence of Type I diabetes per 100000 in 0-34 years old Swedish males and females 1983-1998

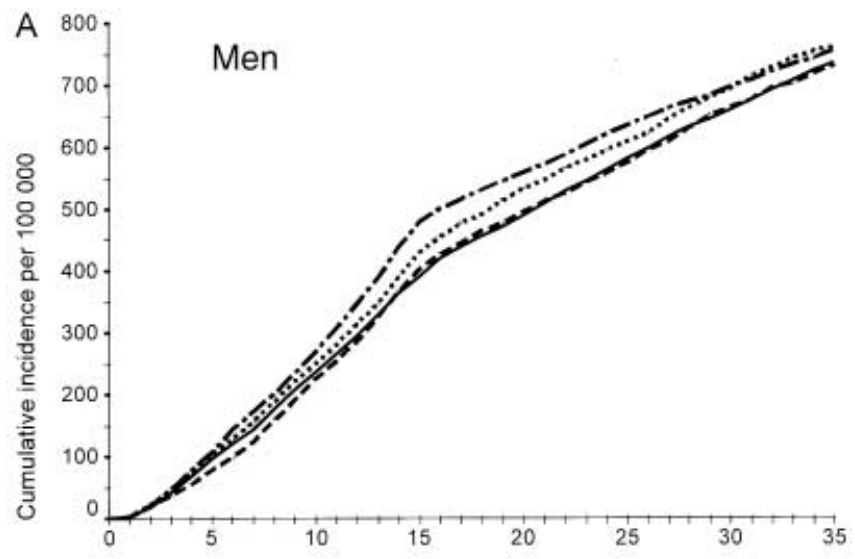

Age at diagnosis (years)

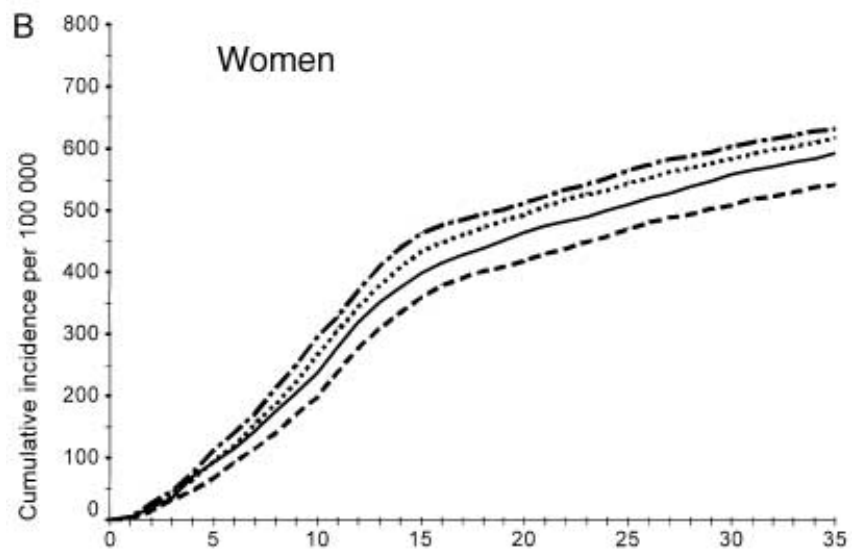

Age at diagnosis (years)

Fig. 4A, B. Cumulative incidence of Type I diabetes per 100000 in 0-34 years old Swedish males (A) and females (B) during four 4-year periods 1983-1998. 1983-86 (-); 1987-90 (- - -); 1991-94 (......); 1995-98 (-..-) 
Table 2. Poisson regression modelling of Type I diabetes incidence in 0-34 years age group in Sweden 1983-1998, with age (3-year groups) and calendar period (4-year periods) as categor- ical variables. Males, 0-2 years age group and 1983-1986 calendar period were used as reference

\begin{tabular}{|c|c|c|c|c|c|c|}
\hline & & \multirow[t]{2}{*}{ Deviance } & \multirow[t]{2}{*}{$d f$} & \multicolumn{3}{|c|}{$\begin{array}{l}\text { Likelihood ratio test } \\
\text { Deviance }\end{array}$} \\
\hline & & & & difference & $d f$ & $p$ value \\
\hline & 1. constant $(\mathrm{c})$ & 4545.0 & 1119 & & & \\
\hline & 2. c, age & 1962.9 & 1108 & 2582.1 & 11 & $<0.001$ \\
\hline & 3. c, age, sex & 1798.6 & 1107 & 164.3 & 1 & $<0.001$ \\
\hline \multirow[t]{4}{*}{ Female } & 5. constant & 2673.6 & 559 & & & \\
\hline & 6. c, age & 748.5 & 548 & 1925.1 & 11 & $<0.001$ \\
\hline & 7. c, age, period & 732.7 & 545 & 15.7 & 3 & 0.001 \\
\hline & 8. c, age, period, age* period & 687.9 & 512 & 44.9 & 33 & 0.08 \\
\hline \multirow[t]{2}{*}{ Male } & 5. constant & 1706.3 & 559 & & & \\
\hline & 6. c, age & 790.2 & 548 & 916.1 & 11 & $<0.001$ \\
\hline
\end{tabular}

age*sex, age*period denote interaction terms between age and sex, and between age and period, respectively

females the cumulative incidence by 15 years of age was lower during 1987-1990, and rose above the value of 1983-1986 during 1991-1994 and 1995-1998, cumulative incidence during the four 4-year periods being 399, 358, 433 and 462, respectively. The same pattern was seen by the 35 years of age $(592,542,617$ and 631). During the last 4-year period (1995-1998), only a slight decrease of rates occurred in the older age groups in females, thus the cumulative incidence was increased both by 15 and 35 years of age.

Poisson regression modelling. Table 2 shows the results of the Poisson regression modelling of age, sex and calendar period effects in the 0-34 years age group. Incidence rates varied significantly depending on the age (model 2) and sex (model 3). The variation of the age-specific incidence rates differed between males and females (model 4), in agreement with Figure 1. Because of the age-sex interaction, the calendar period effect was modelled separately for the sexes. As shown in Table 1, the age-adjusted incidence in the 0-34 years age group did not vary between the 4-year periods in males (model 7). Compared to $1983-1986$ the incidence was $2 \%(p=0.54)$ higher in 1995-1998. However, as can be seen in Figure $2 \mathrm{~A}$, the pattern of change by the time period differed between the 3-year age groups (model 8). In females the age-adjusted incidence in the 0-34 years group varied between the periods (Table 1, model 7). Compared to 1983-1986, the incidence was $8 \%$

Fig. 5A, B. Age-specific incidence of Type I diabetes per 100000 live births in 0-34 years old Swedish males (A) and females (B) born during the 1950s, 1960s, 1970s, 1980s and 1990s (separate line for every birth cohort)

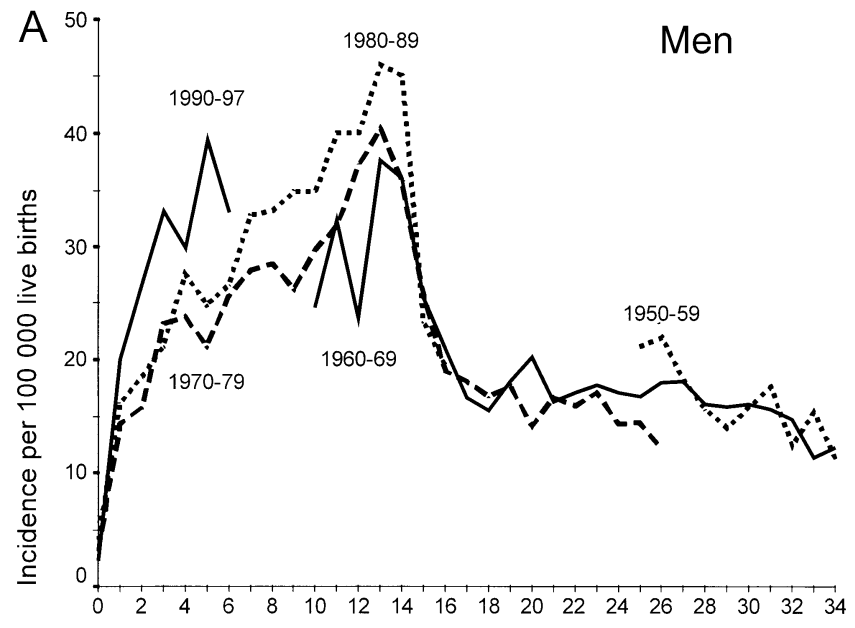

Age at diagnosis (years)

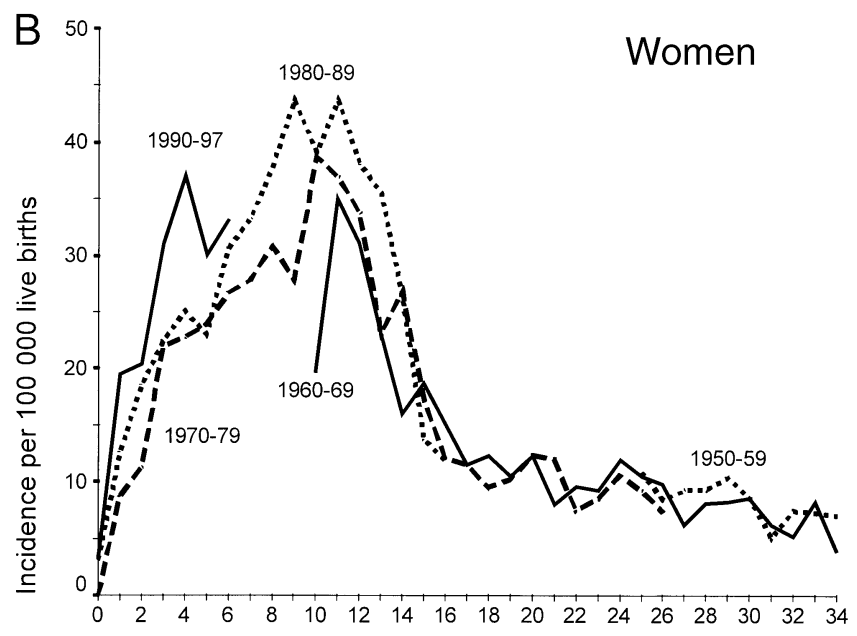

Age at diagnosis (years) 
$(p=0.03)$ lower in 1987-1990, and tended to increase by $7 \%(p=0.10)$ in 1995-1998. The pattern of change by the time period tended to differ between the age groups in females as well, but did not reach statistical significance ( $p=0.08$ ) (Figure 2B, model 8). Thus, no statistically significant increase in incidence has occurred in the 0-34 years group for either sex during the 16-year study period.

Age distribution during four 4-year periods. For both sexes median age at diagnosis in 1983-1986 was higher than in 1995-1998 $(p<0.001)$ (15.0 and 12.5 years in males; 11.9 and 10.4 in females, respectively).

Birth cohort effects. Figure 5 shows incidence rates for 1-year age groups in five birth cohorts, each including 10-year birth intervals, except those born in the 1990s. There has been an increase of age-specific incidence in both boys and girls below 14 years of age at diabetes diagnosis in successive birth cohorts. Compared to children born during the 1970s and 1960s, age-specific incidence was higher for 7-14 years old children born during the 1980s, while for the youngest children, who were 0-6 years of age at diagnosis, incidence increased for the birth cohort of 1990s. However, no clear birth cohort effect could be seen in the older age groups, comparing the birth cohorts of 1950s, 1960s and 1970s.

\section{Discussion}

In this large study, using data from two prospective, population-based diabetes registers with good ascertainment, we found no increase of Type I diabetes incidence in the age group 0 to 34 years in Sweden during a 16-year period. However, median age at diagnosis had decreased. For both sexes, the change of the age-specific incidence over time had opposite directions below and above the age, where the highest agespecific incidence is reached.

Very few studies have analysed time trends of diabetes incidence in the age groups over 14 years at diabetes diagnosis. A tendency of an increase was found in both children (0-14 year) and young adults (15-29 year) in Turin (Italy) 1984 through 1996 [14]. In West Yorkshire, the incidence tended to increase in the $0-14$, and was stable in the 15-29 years age group 1991 through 1997 [15]. However, both of these studies were rather small (817 cases in Turin and 885 in Yorkshire), and thus had a lower power to detect small changes, compared to our study.

There are some methodological issues, important for the interpretation of our results. The completeness of ascertainment of the two diabetes registers differs: it is higher in the SCDS than in the DISS (96-99\% and $86-91 \%$, respectively). There was no clear evidence of a decrease in the completeness of ascertain- ment for Type I diabetes over time in the DISS (see Methods). The completeness of ascertainment during 1992-1997 in the Northern Sweden was the same as during 1983-1987 in the Southern Sweden. However, it was about 10\% lower during 1992-1997 compared to 1986-1991 in the Northern Sweden. Thus, although it is not likely that the tendency of a decrease in incidence seen in the age groups above 14 years of age should be the result of the differences in the case ascertainment only, our results should be interpreted with some caution.

Classification, based on the clinical impression at the time of diagnosis probably underestimates the true number of the Type I diabetes cases, especially in the older age groups, as it is difficult to distinguish the cases with Type II diabetes and LADA from their clinical characteristics [22, 23, 24]. Among the cases, not classified as Type I diabetes at the time of diagnosis in the DISS, about 30\% are ICA-positive [25] and about $50 \%$ are positive for at least one auto-antibody (either ICA, GAD or IA-2A) [24]. Most of these cases probably have Type I diabetes, as $98 \%$ of the ICA-positive and $93 \%$ of those positive for any auto-antibody are treated with insulin six [26] and three [24] years after the diagnosis, respectively. Of the cases registered in the DISS, about $75 \%$ have clear clinical characteristics of Type I diabetes, thus $30-50 \%$ of the remaining cases could be wrongly classified, which would give a 7.5-12.5\% higher incidence of Type I diabetes in the 15-34 years age group. The impact of the misclassification would, however, differ between the age groups, as the proportion of cases, not classified as Type I diabetes increases with age, being $8 \%, 20 \%, 29 \%$ and $42 \%$ in the $15-19,20-24,25-29$ and 30-34 years age groups, respectively [25]. A similar classification based on clinical characteristics was used during the whole study period, and the proportion of cases classified as Type I, as well as the proportion of cases that could not be classified at the time of diagnosis, were stable over time (see Methods). Thus it seems that there has been no major shift in the classification of the type of diabetes over time. However, it should be kept in mind that the completeness of ascertainment for Type II diabetes in the DISS is low (estimated 53\% during 1983-1987) [21], which makes it difficult to assess the true proportion of cases with LADA in the $15-34$ years age group.

Our study indicates that Type I diabetes is not becoming more common, at least not by the age of 35 years in Sweden, but more patients are being diagnosed at an early age. However, it is difficult to estimate how much the results of the current study would be influenced by the inclusion of all cases with LADA in the 15-34 years group, or if the incidence of Type I diabetes was registered even after the age of 34 years. It is not possible to know whether, and to what extent, the observed increase in the incidence of Type I diabetes in childhood is due to a shift towards an earlier age 
at diagnosis, or due to additional cases diagnosed during childhood, or both. If environmental risk factors promoting the disease process, or precipitating the diagnosis by increased insulin demand, have become more prevalent over the years, or the timing of the exposure to such factors has become earlier, this could have led to a clinical presentation of diabetes at an earlier age without increasing the total risk of the disease, a hypothesis which is in agreement with the results of the current study. Adverse changes in insulin sensitivity during the pre-diabetic period are thought to accelerate the rates of progression to frank hyperglycaemia and be important in determining the time of the clinical presentation [27, 28]. Indeed the highest age-specific incidence for both boys and girls in most countries coincides with the timing of puberty and age at peak height velocity, when insulin resistance physiologically increases $[29,30]$. This also explains why the age at peak incidence has changed little over the years although median age at diabetes diagnosis has decreased. However, growth is associated with insulin demand even earlier in childhood. Already in the beginning of 1990's two Swedish case-control studies found that high linear growth rate [31] and weight gain [32] were associated with increased risk of childhood Type I diabetes. More recently higher BMI or relative weight in addition to increased linear growth throughout childhood, but especially during the first three years of life, were shown to be risk factors for the development of Type I diabetes [33, 34, 35]. Thus, increasing height, BMI and prevalence of overweight and obesity in the childhood population over time could have contributed to the observed changes in diabetes incidence. Mean BMI at the age of seven years has increased for both sexes in Stockholm schoolchildren born between 1963 and 1983, without changes in the mean height for the girls, but with an increase in height for the boys [36]. In a large population-based study in Sweden, a higher BMI gain between the age of 2 and 8 years was related to an increased gain in height during the same period, an earlier onset of puberty and less height gain in adolescence [37]. We therefore speculate, that over-nutrition of children could be a part of the explanation of the younger age at diagnosis of Type I diabetes, as it leads to increased growth in height, increased weight gain and BMI during the childhood, as well as an earlier onset of puberty, all associated with increased insulin resistance. Still it cannot be excluded that the activation of the autoimmune process by more, or new triggers has taken place in Sweden.

Our conclusions are based on cumulative incidence, calculated from cross-sectional data during four 4-year periods, and apply to the age span up to 35 years, although Type I diabetes can occur even later in life [6, $7,8,9]$. However, mean BMI and the prevalence of overweight and obesity has increased in the older age groups of both sexes as well [38, 39, 40, 41]. Thus
Type I diabetes cases that would have been diagnosed after 34 years age might be presenting earlier as well, masking the decrease of the incidence in the 15-34 years group. Interestingly, during the 1980s, mean BMI increased most in the 25-34 years age group in Sweden and more for women [38] than for men [39], which could, perhaps, partly explain why the decrease of Type I diabetes incidence was more pronounced in the 15-34 years old males than in females.

Analysis of age-specific incidence rates by birth cohort shows, that childhood Type I diabetes incidence has increased in successive birth cohorts. The most marked increases in incidence seems to have occurred for the 7-14 year-old children born during the 1980 s and for the 0-6 year-old children born during the 1990s. However, no birth cohort extends over the whole age span yet, and the cohort born during the 1980 s could only be followed until the age of 16 years. Thus, it is important to continue the follow-up of these birth cohorts for the confirmation of the results of the present study in a birth cohort material.

Acknowledgements. This work has been supported by grants from the Swedish Medical Research Council (project number 07531), the Swedish Diabetes Association, the Nordic Insulin Foundation and Juvenile Diabetes Foundation - Wallenberg Diabetes Research Program (K98-99JD-128B and K200099JD-12813-O2C). Members of the Swedish Childhood Diabetes Study: Paediatricians L. Skogsberg (Boden), A. Lindh (Borås), K. Segnestam (Eskilstuna), K. Snellman (Falun), A. Stenberg and C. Nilsson (Gällivare), G. KördelEngberg (Gävle), O. Westphal (Göteborg), N.Ö. Nilsson (Halmstad), J. Neiderud (Helsingborg), H. Hörnell and $\AA$. Lagervall (Hudiksvall), L.I. Hardell (Kalmar), H. Edenwall (Karlskrona), G. Jonsell (Karlstad), K. Larsson (Kristianstad), B. Hansing (Lidköping), S. Sjöblad (Lund), S. Ivarsson (Malmö), L. Stenhammar (Norrköping), L. Hellenberg (Nyköping), D. Ström (Skellefteå), M. Bourdin (Skövde), B. Björsell (Sollefteå), T. Torbjörnsdotter (Huddinge), L. Blom (Stockholm), E. Landgren (Sundsvall), R. Hanås (Uddevalla), J. Gustafsson (Uppsala), M. Blomgren (Visby), G. Samuelsson (Trollhättan), B. Eriksson (Västervik), K. Kockum (Ystad), U. Myrdal (Västerås), U. Lindberg (Växjö), U. Ståhle (Ängelholm), J. Åman (Örebro), S. Sjögren (Örnsköldsvik), A.-L. Nilsson (Östersund). Nurses L. Engman (Boden), A. Wiik (Borås), I.-L. Lödesjö (Eskilstuna), J. Dahlgren (Falun), I. Johansson (Gällivare), M. Olsson (Gävle), I. Jansson and M. Hallman (Göteborg), C. Unt (Halmstad), I. Larsson (Helsingborg), L. Kalander (Hudiksvall), B.-M. Weidby (Jönköping), B.-L. Svensson (Kalmar), E. Strigård (Karlskrona), E. Qvist (Karlstad), A. Wallin (Kristianstad), M. Melne (Lidköping), A. Berhagen (Lund), A. Leide (Malmö), G. Hellgren (Norrköping), M. Matiasson (Nyköping), E.-L. Olofsson (Skellefteå), K. Ohlsson (Skövde), M. Henriksson (Sollefteå), M. Selenius (Stockholm), A.-B. Boman (Huddinge), A. Ahlborg-Karlsson and G. Wiklund (Stockholm), K. Svenning (Sundsvall), E. Johansson (Uddevalla), I. Lavin (Umeå), C. Pihlström (Uppsala), M. Grönlund (Visby), D. Bohlin-Fredriksson and C. Ahlberg-Eriksson (Trollhättan), A. Johnsson (Västervik), P. Höglind (Simrishamn), M. Andersson (Västerås), M. Betnér (Växjö), L. Karlsson (Ängelholm), I. Karlsson (Örebro), L. Rönnbäck (Örnsköldsvik), C. Bodén (Östersund), E. Persson (Ystad). 


\section{References}

1. Dahlquist G (1995) Environmental risk factors in human Type 1 diabetes - an epidemiological perspective. Diabetes Metab Rev 11:37-46

2. Åkerblom HK, Knip M (1998) Putative environmental factors in Type 1 diabetes. Diabetes Metab Rev 14:31-67

3. Lernmark $\AA$ (1999) Type 1 diabetes. Clin Chem 45:13311338

4. Atkinson MA, Eisenbarth GS (2001) Type 1 diabetes: new perspectives on disease pathogenesis and treatment. Lancet 358:221-229

5. Freiesleben De Blasio B, Bak P, Pociot F, Karlsen AE, Nerup J (1999) Onset of type 1 diabetes: a dynamical instability. Diabetes 48:1677-1685

6. Nyström L, Dahlquist G, Östman J et al. (1992) Risk of developing insulin-dependent diabetes mellitus (IDDM) before 35 years of age: indications of climatological determinants for age at onset. Int J Epidemiol 21:352358

7. Vandewalle CL, Coeckelberghs MI, De Leeuw IH et al. (1997) Epidemiology, clinical aspects, and biology of IDDM patients under age 40 years. Comparison of data from Antwerp with complete ascertainment with data from Belgium with $40 \%$ ascertainment. The Belgian Diabetes Registry. Diabetes Care 20:1556-1561

8. Laakso M, Pyörälä K (1985) Age of onset and type of diabetes. Diabetes Care 8:114-117

9. Mølbak AG, Christau B, Marner B, Borch-Johnsen K, Nerup J (1994) Incidence of insulin-dependent diabetes mellitus in age groups over 30 years in Denmark. Diabet Med 11:650-655

10. Karvonen M, Tuomilehto J, Libman I, LaPorte R (1993) A review of the recent epidemiological data on the worldwide incidence of Type I (insulin-dependent) diabetes mellitus. World Health Organization DIAMOND Project Group. Diabetologia 36:883-892

11. Onkamo P, Väänänen S, Karvonen M, Tuomilehto J (1999) Worldwide increase in incidence of Type I diabetes the analysis of the data on published incidence trends. Diabetologia 42:1395-1403

12. EURODIAB ACE Study Group (2000) Variation and trends in incidence of childhood diabetes in Europe. Lancet 355:873-876

13. Dahlquist G, Mustonen L (2000) Analysis of 20 years of prospective registration of childhood onset diabetes - time trends and birth cohort effects. Acta Paediatr 89:12311237

14. Bruno G, Merletti F, Biggeri A et al. (2001) Increasing trend of Type I diabetes in children and young adults in the province of Turin (Italy). Analysis of age, period and birth cohort effects from 1984 to 1996. Diabetologia $44: 22-25$

15. McKinney PA, Parslow RC, Feltbower RG, Stephenson C, Bodansky HJ (2000) Rates of Type I diabetes are increasing in children but not young adults in West Yorkshire, UK. Diabetologia 43 [Suppl 1]:A27 (Abstract)

16. Nyström L, Dahlquist G, Rewers M, Wall S (1990) The Swedish childhood diabetes study. An analysis of the temporal variation in diabetes incidence 1978-1987. Int J Epidemiol 19:141-146

17. Dahlquist G, Mustonen L (1994) Childhood onset diabetes - time trends and climatological factors. Int $\mathrm{J}$ Epidemiol 23:1234-1241

18. WHO Expert Committee on Diabetes Mellitus (1980) World Health Organization Technical Report Series No 646, WHO, Geneva
19. World Health Organization (1985) Diabetes mellitus. Report of a WHO Study Group. WHO Technical Report Series No 727, WHO, Geneva

20. Östman J, Arnqvist H, Blohmé G et al. (1986) Epidemiology of diabetes mellitus in Sweden. Results of the first year of a prospective study in the population age group 15-34 years. Acta Med Scand 220:437-445

21. Littorin B, Sundkvist G, Scherstén B et al. (1996) Patient administrative system as a tool to validate the ascertainment in the diabetes incidence study in Sweden (DISS). Diabetes Res Clin Pract 33:129-133

22. Arnqvist HJ, Littorin B, Nyström L et al. (1993) Difficulties in classifying diabetes at presentation in the young adult. Diabet Med 10:606-613

23. Zimmet P, Turner R, McCarty D, Rowley M, Mackay I (1999) Crucial points at diagnosis. Type 2 diabetes or slow type 1 diabetes. Diabetes Care 22 [Suppl 2]:B59-B64

24. Törn C, Landin-Olsson M, Östman J et al. (2000) Glutamic acid decarboxylase antibodies (GADA) is the most important factor for prediction of insulin therapy within 3 years in young adult diabetic patients not classified as Type 1 diabetes on clinical grounds. Diabetes Metab Res Rev 16:442-447

25. Landin-Olsson M, Karlsson FA, Lernmark A, Sundkvist G (1992) Islet cell and thyrogastric antibodies in 633 consecutive 15 - to 34 -yr-old patients in the diabetes incidence study in Sweden. Diabetes 41:1022-1027

26. Littorin B, Sundkvist G, Hagopian W et al. (1999) Islet cell and glutamic acid decarboxylase antibodies present at diagnosis of diabetes predict the need for insulin treatment. A cohort study in young adults whose disease was initially labeled as type 2 or unclassifiable diabetes. Diabetes Care 22:409-412

27. Dahlquist G (1994) Non-genetic risk determinants of type 1 diabetes. Diabetes Metab 20:251-257

28. Leslie RD, Taylor R, Pozzilli P (1997) The role of insulin resistance in the natural history of type 1 diabetes. Diabet Med 14:327-331

29. Amiel SA, Sherwin RS, Simonson DC, Lauritano AA, Tamborlane WV (1986) Impaired insulin action in puberty. A contributing factor to poor glycemic control in adolescents with diabetes. N Engl J Med 315:215-219

30. Moran A, Jacobs DR, Steinberger J et al. (1999) Insulin resistance during puberty: results from clamp studies in 357 children. Diabetes 48:2039-2044

31. Blom L, Persson LÅ, Dahlquist G (1992) A high linear growth is associated with an increased risk of childhood diabetes mellitus. Diabetologia 35:528-533

32. Johansson C, Samuelsson U, Ludvigsson J (1994) A high weight gain early in life is associated with an increased risk of Type I (insulin-dependent) diabetes mellitus. Diabetologia 37:91-94

33. Hyppönen E, Virtanen SM, Kenward MG, Knip M, Åkerblom HK (2000) Obesity, increased linear growth, and risk of type 1 diabetes in children. Diabetes Care 23: 1755-1760

34. Patterson C, Dahlquist G, Soltesz G on behalf of the EURODIAB Substudy 2 Study Group (2001) Early growth and infant feeding and risk of Type I diabetes: a multicentre case-control study. Diabetologia 44 [Suppl 1]:A102 (Abstract)

35. Bruining GJ (2000) Association between infant growth before onset of juvenile type-1 diabetes and autoantibodies to IA-2. Netherlands Kolibrie study group of childhood diabetes Lancet 356:655-656

36. Cernerud L (1993) Height and body mass index of sevenyear-old Stockholm schoolchildren from 1940 to 1990. Acta Paediatr 82:304-305 
37. He Q, Karlberg J (2001) BMI in childhood and its association with height gain, timing of puberty, and final height. Pediatr Res 49:244-251

38. Kuskowska-Wolk A, Bergström R (1993) Trends in body mass index and prevalence of obesity in Swedish women 1980-89. J Epidemiol Community Health 47:195-199

39. Kuskowska-Wolk A, Bergström R (1993) Trends in body mass index and prevalence of obesity in Swedish men 1980-89. J Epidemiol Community Health 47:103-108
40. Rasmussen F, Johansson M, Hansen HO (1999) Trends in overweight and obesity among 18-year-old males in Sweden between 1971 and 1995. Acta Paediatr 88:431-437

41. Rasmussen F, Johansson M (2000) Increase in the prevalence of overweight and obesity from 1995 to 1998 among 18-year-old males in Sweden. Acta Paediatr 89:888889 